\title{
ROHRS' EXAMPLE REVISITED: ON THE ROBUSTNESS OF ADAPTIVE ITERATIVE LEARNING CONTROL
}

\author{
Berk Altın 1 and Kira Barton
}

\begin{abstract}
Adaptive feedback based methods in iterative learning control (ILC) have garnered much interest from researchers for some time now. Much as in adaptive feedback control, most of these methods use Lyapunov functions and positive real transfer functions to prove convergence and boundedness of system signals updated through iterative estimations. While Rohrs et al. have motivated further research on the design of robust adaptive feedback controllers, by demonstrating in the early 1980's that the algorithms of the time were not robust in the presence of unmodeled dynamics, the topic of robustness has not been studied much in the adaptive iterative learning control (AILC) literature. Inspired by Rohrs' counterexample, we use a model reference AILC scheme to show the lack of robustness to unmodeled dynamics in AILC. We rigorously define the concept of stability in ILC via $\mathcal{L}_{2}$ space concepts, and demonstrate the existence of unstable learning operators. We put forth linear systems arguments to explain how conditions leading to instability can occur, and support heuristic arguments with simulation examples. Our findings indicate that the shortcomings of AILC in terms of robustness are no different than those of adaptive feedback, with the robustness issue more severe in certain cases, and further research is necessary to design robust AILC schemes.
\end{abstract}

Key Words: Robustness, learning control, iterative methods, adaptive control, model reference adaptive control.

\section{INTRODUCTION}

Adaptive methods in iterative learning control (ILC) have been a popular area of research in recent years [1]. The main idea is simple, given an uncertain system working on a finite interval repetitively, use estimation schemes in adaptive feedback to iteratively update the input so the control objective is achieved. Much as in adaptive feedback control, most of these methods employ techniques such as the construction of Lyapunov functions and positive real transfer functions to prove convergence and boundedness of the system signals. Special attention has been given to the application area of robotic manipulators, wherein iterative estimation schemes are employed through energy functions $[18,20]$ for improved transient response under parametric uncertainty. Related schemes were also used to reduce the trajectory tracking errors in model reference adaptive control (MRAC)

Manuscript received April 26, 2016; revised April 26, 2017; accepted December $12,2017$.

Berk Altın is with the Department of Computer Engineering, University of California, Santa Cruz, CA, 95064, USA (corresponding author, e-mail: berkaltin@ucsc.edu).

Kira Barton is with the Department of Mechanical Engineering, University of Michigan, Ann Arbor, MI, 48109, USA (e-mail: bartonk1@umich.edu).

The authors would like to thank Enric Xargay for his helpful comments on an earlier draft of the manuscript. This work was conducted while the first author was with the Department of Electrical Engineering and Computer Science at the University of Michigan, and supported by the NSF grant CMMI-1334204.
$[11,20]$. The similarities between adaptive feedback and adaptive iterative learning control (AILC) were further underlined in other works, which have sought to show how adaptive feedback schemes can be extended straightforwardly to ILC to obtain universal learning controllers $[13,16]$. Due to space limitations and the fact that the focus of the current work is adaptive ILC, we refer the readers to the surveys $[1,7,22]$ for a review of recent contributions to the ILC literature.

In the early 1980s, Rohrs et al. demonstrated in their benchmark paper [17] that the adaptive feedback control algorithms of the time were not robust in the presence of unmodeled dynamics. Although it was later argued that the explanation of the instability observed was not adequate $[3,5,6]$, the example constructed in the paper generated much controversy and spurred further research on the design of robust adaptive controllers [15]. Interestingly, despite the prominence of AILC and its similarities with adaptive feedback control, robustness of adaptive iterative learning controllers has not attracted the same level of attention, and is an open question [22]. While several works $[10,21,23]$ have referred to the notion of robustness in AILC, there have been no studies in the literature that tackle the issue of robustness explicitly and in a direct manner. This motivates the question of whether AILC offers benefits in terms of robustness over adaptive feedback, or if it has the same drawbacks as in the feedback case. 
Inspired by this question, in this paper, we use the model reference adaptive ILC (MRAILC) scheme of [19] to demonstrate the lack of robustness of AILC to unmodeled dynamics. The MRAILC scheme of [19] for systems with relative degree 1 is a direct extension of the basic MRAC scheme scrutinized in Rohrs' counterexample, and is therefore a good avenue to explore the convergence properties of AILC in the presence of unmodeled dynamics. Our aim is not to disprove the validity of a specific algorithm, but rather point out through a case study in the spirit of Rohrs' counterexample that AILC is likely to suffer from the same robustness issues as those of adaptive feedback control.

The rest of the paper is organized as follows. Section II generalizes certain function space concepts for ILC systems, followed by a summary of an MRAILC algorithm for systems with relative degree 1 in Section III. The instability of the learning operators of the algorithm are proven in Section IV, with a short discussion of the main assumptions in Section V. We give heuristic linear systems arguments to discuss possible mechanisms of instability and how they can come to occur in Section VI. Simulation results of Section VII are presented to support our arguments, and concluding remarks are given in Section VIII.

\section{NOTATION AND PRELIMINARIES}

We denote by $\mathbb{R}$ the set of real numbers. We take $\mathbb{N}$ as the set of nonnegative integers and $\mathbb{N}^{+}$as the set of positive integers. The space $\mathcal{L}_{2}^{n}$ is the space of Lebesgue measurable functions $f: \mathbb{R} \rightarrow \mathbb{R}^{n}$ with finite norm; i.e.

$$
\|f\|_{\mathcal{L}_{2}} \triangleq\left(\int_{-\infty}^{\infty}\|f(t)\|_{2}^{2} d t\right)^{1 / 2}<\infty
$$

where $\|\cdot\|_{2}$ is the Euclidean norm. Similarly, $l_{2}$ denotes the space of square summable real sequences.

Let $P: \mathcal{V} \rightarrow \mathcal{Y}$ be a mapping where $\mathcal{V}$ is the space of admissible inputs and $\mathcal{Y}$ is the space of outputs. The standard ILC problem is that of finding a controller $C$ that maps the input history $u_{0}, u_{1}, \ldots, u_{k-1} \in$ $V$ to the current input $u_{k} \in V$ such that the output $y_{k}=P u_{k}$ converges to a desired reference $y_{\mathrm{d}}$ in the image of $P$, or a small neighborhood of it, as $k \rightarrow \infty$. $^{*}$ The fact that the map $C$ has the domain $\prod_{k \in \mathbb{N}} \mathcal{V}$ and range $\prod_{k \in \mathbb{N}} \mathcal{Y}$ shows the multidimensionality of the problem setting. In our case, $\mathcal{V}$ and $\mathcal{Y}$ will

\footnotetext{
* Here, we are assuming that $P$ is known and there are no exogenous inputs affecting the error, so that any function of the error can be transformed into a function of the input by substituting $y_{\mathrm{d}}-P u_{k}$. Thus, it is sufficient to consider the sequence of inputs.
}

be $\mathcal{L}_{2}$ spaces of different dimensions on a finite interval. Hence, a signal $f$ will be defined as a function that maps a given iteration $k$ and time $t$ to an $n$ dimensional real vector. This motivates us to define the concepts of stability and gain for mappings such as $C$. We introduce several definitions to rigorously formulate these for ILC systems.

Definition $1\left(\mathcal{L} l_{2}\right.$ space). $\mathcal{L} l_{2}^{n}$ is the space of all function sequences $f=\left(f_{0}, f_{1}, \ldots\right)$, where $f_{k}: \mathbb{R} \rightarrow \mathbb{R}^{n}$ is Lebesgue measurable for all $k \in \mathbb{N}$, and the $\mathcal{L} l_{2}$ norm of $f$ given by

$$
\|f\|_{\mathcal{L l}_{2}} \triangleq\left(\sum_{k=0}^{\infty}\left\|f_{k}\right\|_{\mathcal{L}_{2}}^{2}\right)^{1 / 2}
$$

is finite.

Definition 2 (Extended $\mathcal{L} l_{2}$ space). The extended space $\mathcal{L} l_{2 e}^{n}$ is the space of all $f$ such that $(f)_{\kappa} \in \mathcal{L} l_{2}^{n}$ for all $\kappa \in \mathbb{N}$, where $(f)_{\kappa} \triangleq\left(f_{0}, f_{1}, \ldots, f_{k}, 0,0, \ldots\right)$ is the truncation of $f$.

The idea of the extended space as defined above may seem redundant due to the discrete nature of the problem setting, as a sequence $f \in \mathcal{L} l_{2 e}$ if and only if every $f_{k} \in \mathcal{L}_{2}$. Moreover, if each $f_{k}$ has bounded support, the condition is automatically satisfied. Nevertheless, it will enable us to focus on the convergence properties of the iterative problem rather than the time domain dynamics.

Definition 3 (Finite gain $\mathcal{L} l_{2}$ stability). A mapping $P$ : $\mathcal{L} l_{2 e}^{m} \rightarrow \mathcal{L} l_{2 e}^{n}$ is finite gain $\mathcal{L} l_{2}$ stable if there exist finite constants $\alpha$ and $\beta$ such that

$$
\left\|(P u)_{K}\right\|_{\mathcal{L}_{2}} \leq \alpha\left\|(u)_{\kappa}\right\|_{\mathcal{L l}_{2}}+\beta \quad \forall u \in \mathcal{L} l_{2 e}^{m}, \kappa \in \mathbb{N}
$$

Otherwise, $P$ is said to have infinite gain or be unstable.

In addition to the above, we will also say that $f$ is bounded if there exists a finite constant $M$ such that $\left\|f_{k}(t)\right\|_{\infty} \leq M$ for all $k \in \mathbb{N}$ and $t \in \mathbb{R}$, where $\|\cdot\|_{\infty}$ is the sup norm, and unbounded otherwise. The definitions above are natural generalizations of the classical $\mathcal{L}_{2}$ space concepts to iterative signals: If $\|f\|_{\mathcal{L}_{2}}=\epsilon, f$ has a total energy of $\epsilon^{2}$. We have chosen the notation $\mathcal{L} l_{2}$ to reflect the two dimensional nature of iterative signals as each $f_{k} \in \mathcal{L}_{2}$ and $\left(\left\|f_{0}\right\|_{\mathcal{L}_{2}},\left\|f_{1}\right\|_{\mathcal{L}_{2}}, \ldots\right)$ is an $l_{2}$ sequence. For our discussion, we will assume that each signal $f_{k}$ has the same bounded support, namely the finite interval $[0, T]$ as per the ILC assumption. Note that the truncation operator is defined on the iteration domain since we are interested in this axis. 
Finally, let $\left(E_{1}, E_{2}, \ldots, E_{n}\right)$ be the standard basis of $\mathbb{R}^{n}$. We say that $f \in \mathcal{L} l_{2 e}^{n}$ has an iteration-invariant component if there exist $m \in\{1,2, \ldots, n\}$ such that

$$
E_{m}^{\top} f_{k}(t)=E_{m}^{\top} f_{k+1}(t) \quad \forall k \in \mathbb{N}, t \in[0, T]
$$

Furthermore, the sequence $f^{\prime} \triangleq\left(f_{0}^{\prime}, f_{1}^{\prime}, \ldots\right)$, where $f_{k}^{\prime}(t) \triangleq E_{m}^{\top} f_{k}(t)$ for all $k \in \mathbb{N}$ and $t \in[0, T]$, is called an iteration-invariant component of $f$. With some abuse of notation, we will also denote by $f^{\prime}$ the function $f_{0}^{\prime}$. Similarly, we say that $f$ is iteration-invariant if (1) holds for all $m \in\{1,2, \ldots, n\}$.

\section{PROBLEM SETUP}

In this section, we introduce the MRAILC algorithm of [19] for plants with relative degree 1 . We will limit the discussion to this algorithm for simplicity and consistency with Rohrs' example. The algorithm is a direct extension of the standard unnormalized MRAC algorithm for plants with relative degree 1 . In addition, we will introduce the error model structure of the algorithm, which will be used later to argue how instability can arise.

\subsection{The MRAILC algorithm for relative degree 1 systems}

Consider a nominal single-input single-output (SISO) linear time-invariant (LTI) minimum phase plant

$$
G_{\mathrm{P}}(s)=g_{\mathrm{P}} \frac{Z_{\mathrm{P}}(s)}{R_{\mathrm{P}}(s)}
$$

with relative degree 1 , where $g_{\mathrm{P}} \in \mathbb{R} \backslash\{0\}$ is the high-frequency gain, and $Z_{\mathrm{P}}(s)$ and $R_{\mathrm{M}}(s)$ are monic polynomials. The nominal plant $G_{\mathrm{P}}(s)$ is unknown, but it is assumed that an upper bound $n$ on the degree of $R_{\mathrm{P}}(s)$ and the sign of $g_{\mathrm{P}}$ is known. Similarly, consider a SISO LTI strictly positive real (SPR) reference model

$$
G_{\mathrm{M}}(s)=g_{\mathrm{M}} \frac{Z_{\mathrm{M}}(s)}{R_{\mathrm{M}}(s)},
$$

where $g_{\mathrm{M}} \in \mathbb{R} \backslash\{0\}$ is the high-frequency gain, and $Z_{\mathrm{M}}(s)$ and $R_{\mathrm{M}}(s)$ are monic Hurwitz polynomials with degree less than $n$. The reference model $G_{\mathrm{M}}(s)$ has relative degree 1 , and can be designed to ensure desired closed-loop dynamics. The control law of the algorithm is given by

$$
u_{k}(t)=\theta_{k}^{\top}(t) \Omega_{k}(t) \quad \forall k \in \mathbb{N}, t \in[0, T]
$$

where $\theta_{k}(t) \in \mathbb{R}^{2 n}$ is the output of the parametric adaptation law, and $\Omega_{k}(t) \in \mathbb{R}^{2 n}$ is the vector of measured variables containing the reference $r(t) \in \mathbb{R}$, along with filtered copies of the input $u_{k}(t) \in \mathbb{R}$ and output $y_{k}(t) \in$ $\mathbb{R}$. Specifically, $\Omega_{k}(t)=\left[y_{k}(t) r(t)\right]^{\top}$ for all $k \in \mathbb{N}$ and $t \in[0, T]$ when $n=1$, and using the standard mixed notation,

$$
\Omega_{k}(t)=\left[\begin{array}{c}
\frac{\alpha(s)}{\Lambda_{0}(s) Z_{\mathrm{M}}(s)}\left[u_{k}(t)\right] \\
\frac{\alpha(s)}{\Lambda_{0}(s) Z_{\mathrm{M}}(s)}\left[y_{k}(t)\right] \\
y_{k}(t) \\
r(t)
\end{array}\right] \quad \forall k \in \mathbb{N}, t \in[0, T],
$$

when $n \geq 2$, where $\alpha(s)=\left[s^{n-2} s^{n-3} \ldots 1\right]^{\top}$ and $\Lambda_{0}(s)$ is an arbitrary polynomial such that $\Lambda_{0}(s) Z_{\mathrm{M}}(s)$ is a Hurwitz polynomial of degree $n-1$. The adaptation law is defined as

$$
\begin{aligned}
& \dot{\theta}_{0}(t)=\Gamma \Omega_{0}(t) e_{0}(t) \operatorname{sgn}\left(p^{*}\right) \\
& \theta_{k}(t)=\theta_{k-1}(t)+\Gamma \Omega_{k}(t) e_{k}(t) \operatorname{sgn}\left(p^{*}\right)
\end{aligned}
$$

for all $k \in \mathbb{N}^{+}$and $t \in[0, T]$, where $\Gamma \in \mathbb{R}^{2 n \times 2 n}$ is the symmetric positive definite adaptation gain matrix, $e_{k}(t) \triangleq y_{\mathrm{d}}(t)-y_{k}(t)$ is the output tracking error with $y_{\mathrm{d}}(t) \in \mathbb{R}$ the desired trajectory at time $t$, and the constant $p^{*}=g_{\mathrm{P}} / g_{\mathrm{M}}$ is the ratio of the high-frequency gains of the nominal open-loop plant and the reference model. Note that for $k=0$, the controller parameter vector and the input are computed using the standard MRAC algorithm over the finite time interval. The dynamics of the MRAILC algorithm in the iteration domain with the uncertain open-loop plant $G(s)$, under the influence of external disturbances, is shown in Fig. 1, where it is assumed that $p^{*}>0$. Without loss of generality, in the sequel, we suppose $p^{*}>0$.

\subsection{Error model structure of the algorithm}

The error signal of the classical MRAC algorithm for relative degree 1 plants is given by

$$
e(t)=\left(G^{*}(s)-G_{\mathrm{M}}(s)\right)[r(t)]+G^{*}(s)\left[\frac{\tilde{\theta}^{\top}(t) \Omega(t)}{\theta_{r}^{*}}\right]
$$

for all $t \in[0, T]$. We denote by $\theta^{*} \in \mathbb{R}^{2 n}$ the constant vector that achieves $G^{*}(s)=G_{\mathrm{M}}(s)$ when the relative degree of $G_{\mathrm{P}}(s)$ is 1 , where $G^{*}(s)$ is the closed-loop transfer function that would result with $u(t)=\left(\theta^{*}\right)^{\top} \Omega(t)$ for all $t \geq 0$. Here, $\tilde{\theta}(t) \triangleq \theta(t)-\theta^{*}$ is the parameter estimation error, and $\theta_{r}^{*}$ is the component of $\theta^{*}$ that acts on $r(t)$. Hence, 


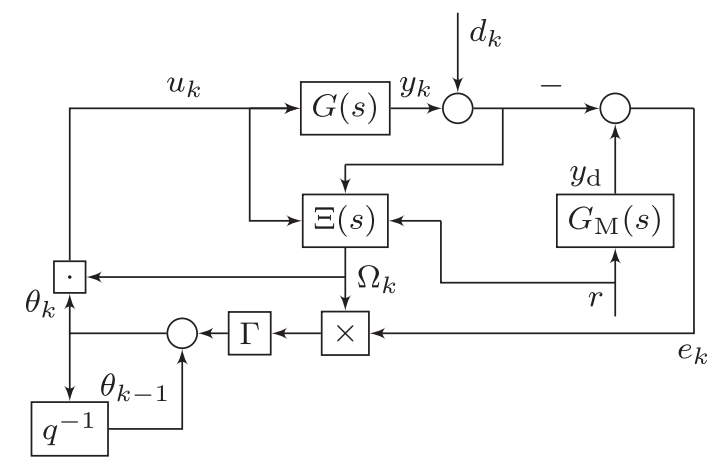

Fig. 1. Block diagram of the MRAILC agorithm with the forward trial-shift operator $q$, where it is assumed that $p^{*}>0$, and "." denotes the dot product. The filter $\Xi(s)$ depends on a Hurwitz polynomial of choice.

the error signal of the MRAILC algorithm is

$$
e_{k}(t)=\left(G^{*}(s)-G_{\mathrm{M}}(s)\right)[r(t)]+G^{*}(s)\left[\frac{\tilde{\theta}_{k}^{\top}(t) \Omega_{k}(t)}{\theta_{r}^{*}}\right]
$$

for all $k \in \mathbb{N}^{+}$and $t \in[0, T]$. As stated in [17], if the restrictive relative degree assumption is violated, $G^{*}(s)$ can be as close to $G_{\mathrm{M}}(s)$ as the feedback structure allows.

\section{INSTABILITY OF THE LEARNING OPERATORS}

We will now analyze the MRAILC algorithm given by (2) and (3) in the same quantitative manner of [17]. This approach will enable us to later use linear systems arguments to discuss how the conditions bringing instability could be introduced to the system by exogenous signals. Since we are interested in the convergence properties on the iteration axis, we can treat $\theta_{0}$ as an initial condition to rewrite the estimation as a pointwise integrator;

$$
\theta_{k}(t)=\theta_{0}(t)+\Gamma \sum_{l=1}^{k} \Omega_{l}(t) e_{l}(t)
$$

for all $k \in \mathbb{N}^{+}$and $t \in[0, T]$. Consequently, the control at the $k$ th iteration is given as

$$
u_{k}(t)=\left(\theta_{0}(t)+\Gamma \sum_{l=1}^{k} \Omega_{l}(t) e_{l}(t)\right)^{\top} \Omega_{k}(t)
$$

for all $k \in \mathbb{N}^{+}$and $t \in[0, T]$. Now let

$$
\begin{gathered}
\Omega \triangleq\left(\Omega_{0}, \Omega_{1}, \ldots\right), \quad \theta \triangleq\left(\theta_{0}, \theta_{1}, \ldots\right), \\
e \triangleq\left(e_{0}, e_{1}, \ldots\right), \quad u \triangleq\left(u_{0}, u_{1}, \ldots\right) .
\end{gathered}
$$

We define $H_{\Omega}: \mathcal{L} l_{2 e} \rightarrow \mathcal{L} l_{2 e}^{2 n}$ as the operator mapping $e$ to $\theta$ according to (5), and $G_{\Omega}: \mathcal{L} l_{2 e} \rightarrow \mathcal{L} l_{2 e}$ as the mapping from $e$ to $u$ according to (6), both parametrized by $\Omega$. It is trivial to see that there exists $\Omega$ such that both operators have infinite gain when isolated from the rest of the system.

Theorem 1. If $\Omega$ has an iteration-invariant component $w$ such that $w(t) \triangleq b+c \sin \left(\omega_{0} t\right)=E_{m}^{\top} \Omega_{0}$ for all $t \in[0, T]$ for some $m \in\{1,2, \ldots, 2 n\}$ and positive constants $b, c$, and $\omega_{0}$, then $H_{\Omega}$ has infinite gain.

Proof. We prove this by construction as in [17]. Without loss of generality, assume $\Gamma$ to be the identity matrix and $\theta_{0}=0, e_{0}=0$. Let $e_{k}(t)=a \sin \left(\omega_{0} t+\phi\right)$ for all $t \in[0, T]$ and $k \in \mathbb{N}^{+}$. It suffices to consider the component of the vector $\theta_{k}$ that arises due to $w$. Hence, we denote this component $\varphi_{k}, \varphi_{k}(t) \triangleq \sum_{l=0}^{k} w(t) e_{l}(t)$. Since $e_{0}=0,\left\|\varphi_{k}\right\|_{\mathcal{L}_{2}}=k \gamma$ for some finite constant $\gamma$. Now let $\varphi=\left(\varphi_{0}, \varphi_{1}, \ldots\right)$. Then we have

$$
\left\|(\varphi)_{\kappa}\right\|_{\mathcal{L}_{2}}=\gamma\left(\sum_{l=1}^{\kappa} l^{2}\right)^{1 / 2}=\gamma \sqrt{\frac{\kappa(\kappa+1)(2 \kappa+1)}{6}},
$$

by Faulhaber's formula. Since $\left\|(e)_{\kappa}\right\|_{\mathcal{L}_{2}}=\sqrt{\kappa}\left\|e_{1}\right\|_{\mathcal{L}_{2}}$, from (7)

$$
\frac{\left\|(\varphi)_{\kappa}\right\|_{\mathcal{L} l_{2}}}{\left\|(e)_{K}\right\|_{\mathcal{L} l_{2}}}=\sqrt{\frac{(\kappa+1)(2 \kappa+1)}{6}} \frac{\gamma}{\left\|e_{1}\right\|_{\mathcal{L}_{2}}},
$$

so $\lim _{\kappa \rightarrow \infty} \frac{\left\|(\varphi)_{\kappa}\right\|_{\mathcal{L}_{2}}}{\left\|(e)_{\kappa}\right\|_{\mathcal{L}_{2}}}=\infty$. Thus, $H_{\Omega}$ has infinite gain.

It is clear from the definition of $\varphi_{k}$ that $H_{\Omega}$, when parametrized by the bounded vector $\Omega$, is bounded-input bounded-output unstable in the sense that a bounded input $e$ forces the output $\varphi$ to grow unboundedly. Also observe that as opposed to the quantitative proof of [17], the proof of instability is much less tedious in the iterative case as a result of the iteration invariance assumption. We discuss this assumption in the next section. For completeness, we also state the instability of $G_{\Omega}$. 
Theorem 2. If $\Omega$ has an iteration-invariant component $w$ such that $w(t) \triangleq b+c \sin \left(\omega_{0} t\right)=E_{m}^{\top} \Omega_{0}$ for all $t \in[0, T]$ for some $m \in\{1,2, \ldots, 2 n\}$ and positive constants $b, c$, and $\omega_{0}$, then $G_{\Omega}$ has infinite gain.

Proof. Let $\theta_{0}=0$ and choose $e$ as in the proof of Theorem 1. Assume $\Omega_{0}=\left(w_{1}, w_{2}, \ldots, w_{2 n}\right)$ is such that $w_{i}=w$ for some $i \in\{1,2, \ldots, 2 n\}$. Then, $\left|u_{k}(t)\right| \geq$ $k\left|e_{1}(t)\right| w^{2}(t)$ for all $k \in \mathbb{N}^{+}$and $t \in[0, T]$. The rest of the proof follows the same steps as before.

\section{ASSUMPTION OF ITERATION-INVARIANT SINUSOIDALS}

The central argument of the proofs of instability is that $\left\|(\varphi)_{\kappa}\right\|_{\mathcal{L l}_{2}}$ (or $\left\|(u)_{\kappa}\right\|_{\mathcal{L l}_{2}}$ ) is $O\left(\kappa^{3 / 2}\right)$, while $\left\|(e)_{\kappa}\right\|_{\mathcal{L} l_{2}}$ is $O\left(\kappa^{1 / 2}\right)$, which is due to the iteration-invariance of $w$ and $e$. We explain our reasoning for this assumption as follows: In [17], the authors use LTI systems arguments to heuristically argue that closed-loop stability can be violated under certain conditions when unmodeled high-frequency time-domain dynamics are present. Hence, sinusoidal error and parameter signals are used to show the instability of the feedback operators in the time domain. By the same token, we assume the same sinusoidal structure for the error and parameter signals. On the other hand, the assumption of iteration invariance relies on the fact that if an exogenous signal exists to induce sinusoidal behavior in $e_{k}$ and $w$ (e.g. $60 \mathrm{~Hz}$ hum, or a sinusoidal reference) for some $k \in \mathbb{N}$, it is likely to persist up to a phase shift in the following iterations. This assumption can be relaxed in many ways. For instance, if $e_{k}$ is bounded and $\Omega_{k}$ has a component $w_{k}$ such that $e_{k}$ and $w_{k}$ are both bounded away from 0 in an interval contained in $[0, T] \forall k \geq K$, for some $K \in \mathbb{N}^{+}$, the proof follows in a similar manner: By

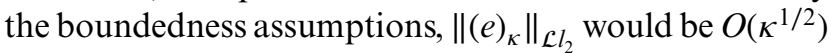
and $\left\|(\varphi)_{\kappa}\right\|_{\mathcal{L l}_{2}}$ would be bounded from below by an $O\left(\kappa^{3 / 2}\right)$ function.

\section{MECHANISMS OF INSTABILITY}

It is discussed in [5,6] that the arguments of [17] do not capture all aspects of the problem. In $[5,6]$, the author uses averaging methods to further analyze Rohrs' counterexample. It is shown that since a step input is persistently exciting of order 1 , the set of equlibria of the parameters is an affine subspace of $\mathbb{R}^{2}$ instead of an isolated point. Thus, parameters can drift on this line, thereby leaving the region where the linearized system is stable. Hence, controller parameters cannot be deter- mined reliably in the presence of unmodeled dynamics and disturbances. Similarly, outside the bandwidth of the plant where unmodeled dynamics are present, phase shifts of $\pm 180^{\circ}$ can occur, thereby violating the positive realness condition.

The iterative adaptation law (3) manifests as an unstable linear discrete "time" (iteration)-varying system for each $t \in[0, T]$, so it may be argued that similar arguments can be made for the adaptive iterative controller. However, it is not straightforward how time-domain dynamics can be related to this pointwise integrator to show that the equilibrium set of $\theta_{k}$ has possibly more than a single element, ${ }^{\dagger}$ as the the system state $\theta_{k}$ lies in an infinite-dimensional subspace of $\mathcal{L}_{2}$. Nevertheless, linear systems concepts can be used in the style of [17] to give a sufficiently intuitive explanation of how instability can occur. Let us now try to give heuristic explanations in the same way.

\subsection{Existence of the sinusoidals}

We argued in Section $\mathrm{V}$ that if an exogenous signal exists to induce sinusoidal behavior, it is likely to be invariant over the iteration domain. This argument is consistent with the assumptions of classical ILC, and also that of [17]. For example, the invariance of the reference (or disturbance) from trial-to-trial is a standard assumption in ILC. On the other hand, certain disturbances that affect system dynamics during a trial, such as measurement noise, load disturbance, or mains hum, will also persist in future trials. Thus, we can consider a single trial in conjecturing how these signals can be introduced to the feedback system.

First, assume that the reference signal is sinusoidal with frequency $\omega_{0}$ and a bias term. Then, from linear systems theory, the output $y_{k}$, and consequently all components of $\Omega_{k}$, will be sinusoidals at frequency $\omega_{0}$ with a bias term and a phase shift. Now under the common assumption that plant uncertainties occur at high frequencies, $G^{*}(s)$ will match $G_{\mathrm{M}}(s)$ at DC, but not at a large enough $\omega_{0}$. Therefore, $e_{k}$ will be a sine wave at frequency $\omega_{0}$. Thus, the conditions for the infinite gains of $G_{\Omega}$ and $H_{\Omega}$ will be satisfied.

\footnotetext{
$\dagger$ In fact, it is trivial to see via the Laplace transform that in an infinite horizon setting (i.e. $T=\infty$ ) there is a unique time-invariant parameter signal for all nonzero references achieving perfect tracking. In other words, assuming uniform convergence to 0 , any nonzero reference is sufficiently rich of all orders in the iterative domain due to the fact that the signal space $\mathcal{L}_{2}$ is infinite dimensional. For instance, when the reference is a step, perfect tracking implies that the closed-loop system must match the whole spectrum of the reference model as opposed to just zero frequency (DC), which is the case in adaptive feedback. Now the question is whether we can find a time-varying parameter that can also achieve perfect tracking given a nonzero reference.
} 
Now we suppose that a sinusoidal disturbance $d$ of frequency $\omega_{0}$ corrupts the output $y_{k}$ additively. Then, the measured output $y_{k}+d$ will have a sinusoid at frequency $\omega_{0}$, and so will $\Omega_{k}$ and $e_{k}$ by definition. Again, the conditions for infinite gain will be satisfied.

\subsection{Destabilizing effects of the infinite-gain operators}

In [19], the author considers an extension of the well known SPR-Lyapunov design approach [14], coined Iterative-SPR-Lyapunov lemma. Under the assumption that $G_{\mathrm{P}}(s)$ has relative degree $1, G^{*}(s)$ can be made equal to $G_{\mathrm{M}}(s)$, simplifying (4) to

$$
e_{k}(t)=G^{*}(s)\left[\frac{\tilde{\theta}_{k}^{\top}(t) \Omega_{k}(t)}{\theta_{r}^{*}}\right] \quad \forall k \in \mathbb{N}, t \in[0, T] .
$$

Since $G^{*}(s)$ is SPR, the system is known to be stable for $k=0$. In addition, for each $t \in[0, T]$, the SPR assumption enables the construction of a discrete Lyapunov functional over the iteration domain that is nonincreasing, thereby proving pointwise convergence of the error to 0 and boundedness of all signals.

We consider two pathological cases that may lead to the failure of this approach. First, the fact that the error converges pointwise to 0 is a key ingredient of the MRAILC algorithm of Section III, since the converse implies a divergent series. If there is a steady-state error on an interval contained in $[0, T]$, caused by a persistent disturbance, the adaptive law will integrate this error with each iteration, and $\theta$ will be unbounded by virtue of the infinite gain of $H_{\Omega}$. For the second case, we assume that $\omega_{0}$ is large enough so that $G^{*}(s)$ is subject to phase shifts of $\pm 180^{\circ}$ when unmodeled dynamics are present. To analyze the case, we direct our attention to Fig. 2 and note that the error system of the MRAILC is a feedback controller in the iteration domain. Thus, the phase shift of $\pm 180^{\circ}$ combined with the infinite gain will affect the iterative loop the same way it affects the feedback loop, by enforcing the error signal so it grows in amplitude with each iteration. In other words, if the relative degree assumption is violated so that $G^{*}(s)$ does not equal $G_{\mathrm{M}}(s)$, and $G^{*}(s)$ has a $\pm 180^{\circ}$ phase shift, the iterative controller will create a positive feedback loop due to a change of sign that will force signals to grow unboundedly.

It is well worth noting that these results are to be expected: The MRAILC algorithm can be interpreted as a standard MRAC scheme where the exogenous signals are periodic with period $T$, and the adaptation law is reformulated as a periodic update. Therefore, it should not come as a surprise that the MRAILC system loses

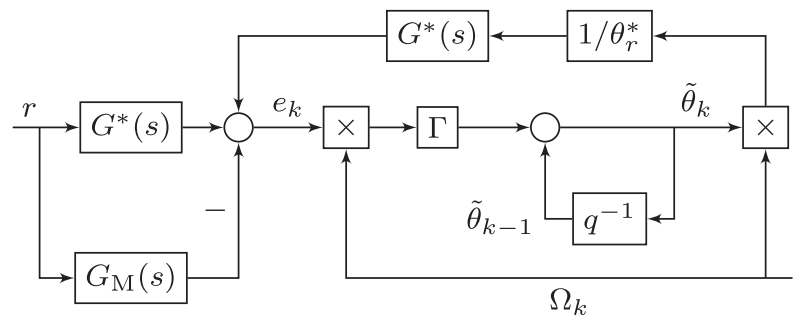

Fig. 2. Error model of the MRAILC with the forward trial-shift operator $q$.

stability in the same conditions as MRAC. We verify these via simulation in the following section.

\section{SIMULATIONS}

The heuristic arguments of the previous section provide good intuition into causes of instability, but do not necessarily describe the situation accurately since the adaptive system is nonlinear. Hence, in this section we will present several simulation scenarios to support our claims. We consider the following model that was originally presented in [17]:

$$
\begin{aligned}
G_{\mathrm{P}}(s) & =\frac{2}{s+1}, & G(s) & =\frac{2}{s+1} \frac{229}{s^{2}+30 s+229}, \\
G_{\mathrm{M}}(s) & =\frac{3}{s+3}, & \Omega_{k}(t) & =\left[r(t) y_{k}(t)\right]^{\top} .
\end{aligned}
$$

We define $\omega_{k}^{r}(t)$ and $\omega_{k}^{y}(t)$ such that

$$
\theta_{k}(t)=\left[\omega_{k}^{r}(t) \omega_{k}^{y}(t)\right]^{\top},
$$

and initialize the simulations with

$$
\theta_{0}(0)=[1.14-0.65]^{\top},
$$

thereby yielding the stable system

$$
G^{*}(s)=\frac{527}{s^{3}+31 s^{2}+259 s+527}
$$

for $\theta^{*}=\theta_{0}(0)$. We take the adaptation rate $\Gamma$ to be the identity matrix. We choose $T$ to be relatively small at 5 seconds to ensure "stability" in the time domain and that signals remain bounded for a few iterations. Note that since the adaptation law is static (therefore causal) in time, the short simulation time does not affect the signal content, in the sense that taking $T>5$ would have resulted in the same signals for the first 5 seconds of each iteration. We also note that when $G(s)$ is equal to $2 /(s+1)$, i.e. there are no unmodeled dynamics, the MRAILC algorithm guarantees pointwise 


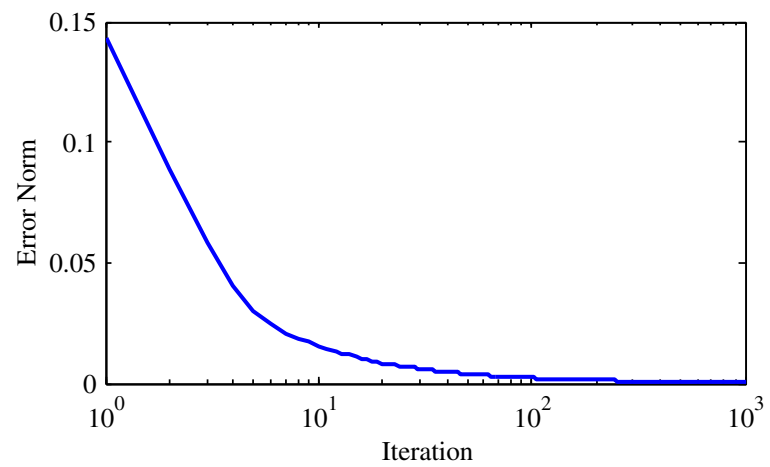

Fig. 3. Stable learning of a sinusoidal reference under perfect modeling without disturbance; the desired reference is $r(t)=0.3+2 \sin (4 t)$. [Color figure can be viewed at wileyonlinelibrary.com].

convergence to 0 for $G_{\mathrm{P}}(s), G_{\mathrm{M}}(s), \Omega_{k}(t), \theta_{k}(t), \Gamma$ chosen as above.

For our first example, we will consider a sinusoidal reference. In [17], it was shown that the system went unstable for $r(t)=0.3+1.85 \sin (16.1 t)$, where $16.1 \mathrm{rad} / \mathrm{s}$ is the frequency at which $G(s)$ has a $180^{\circ}$ phase shift. On the other hand, all closed-loop signals were shown to remain bounded for $r(t)=0.3+2 \sin (8 t)$, despite a "bursting" period. Hence, we take a more modest frequency of 4 $\mathrm{rad} / \mathrm{s}$ and simulate the system for $r(t)=0.3+2 \sin (4 t)$. We observe in Fig. 3 that when unmodeled dynamics are not present, i.e. $G(s)$ equals $G_{\mathrm{M}}(s)$, the MRAILC algorithm seems to drive the error to 0 in the $\mathcal{L}_{2}$ norm topology despite the fact that only pointwise convergence was proven. On the other hand, Fig. 4 indicates that while the MRAC scheme (trial 0 ) remains stable for 5 seconds, the resulting tracking error at trial 8 is larger than the original error. In fact, our simulation had a singularity at the attempted 9th trial, with an infinite-derivative error. A closer look at the evolution of the parameter vector reveals the infinite-gain action of $H_{\Omega}$, where the final parameter signal can be seen to be much larger than the initial. We also note that the parameter has evolved in a complicated manner and the signal at the 8 th iteration is rich with harmonics. The infinite-gain action that leads to stability is perhaps most easily seen in the last graph, where we see that the MRAILC scheme indeed reduces the $\mathcal{L}_{2}$ norm of the tracking error for 2 iterations before going unstable.

For the second case, we consider the problem of setpoint tracking with $r(t)=1.0$. Although not shown here, the MRAC scheme performs quite well with very stable behavior and perfect tracking for prolonged periods of time since $G^{*}(s)$ readily matches $G(s)$ at DC. However, Fig. 5 shows that the error again starts to grow (a)

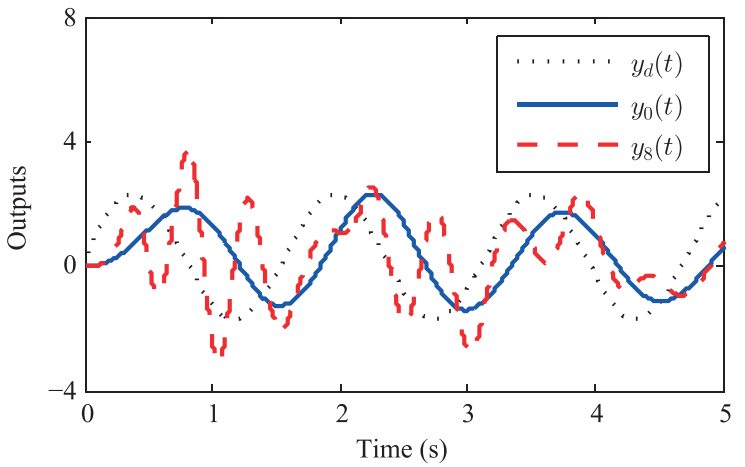

(b)

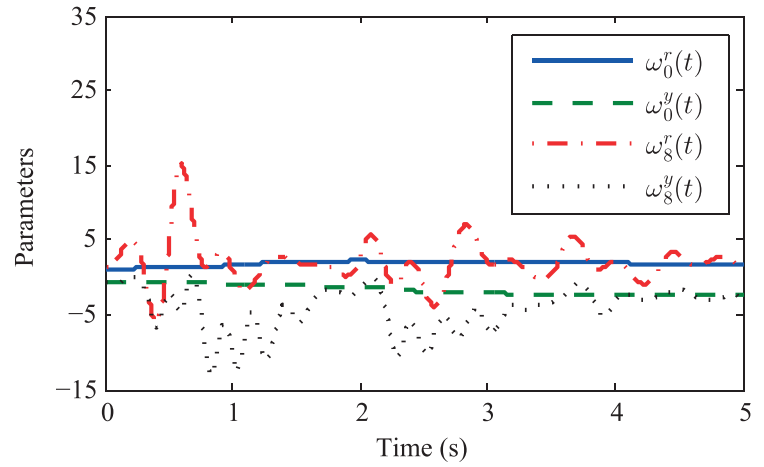

(c)

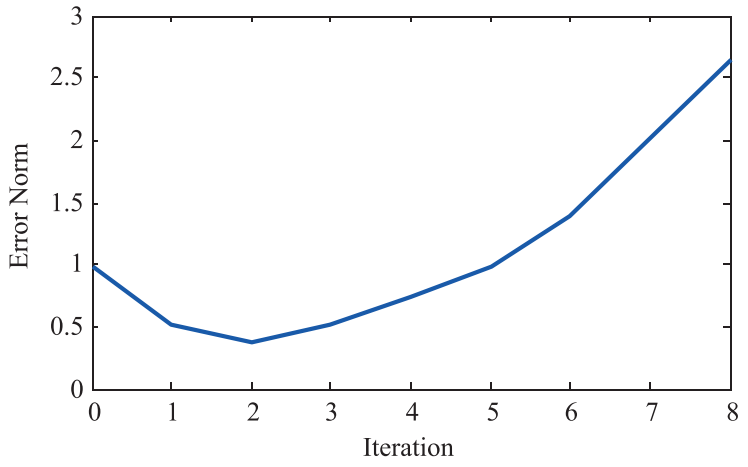

Fig. 4. Instability in sinusoidal reference tracking without disturbance; the desired reference is $r(t)=0.3+2 \sin (4 t)$. (a) Evolution of the output from the first to the last iteration, (b) Evolution of the parameter vector from the first to the last iteration and (c) Evolution of the error $\mathcal{L}_{2}$ norm in the iteration domain. [Color figure can be viewed at wileyonlinelibrary.com]

unboundedly with each trial after a reduction period of 3 trials. Hence, we see that while the MRAC scheme offers high performance in DC, an attempt to improve the transients leads to instability in the iteration domain. In this case, an appropriate takeaway message would be that the MRAILC fails to apply the "common-sense rule" of not fitting a model to bad data [4]: Although system 
(a)

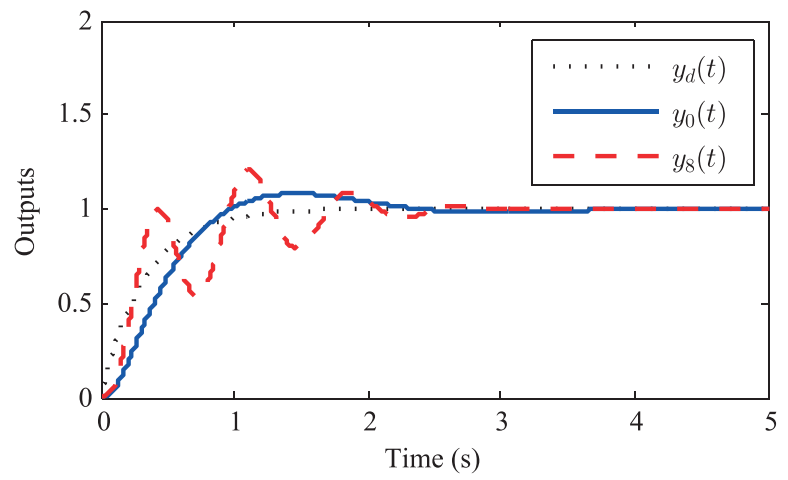

(b)

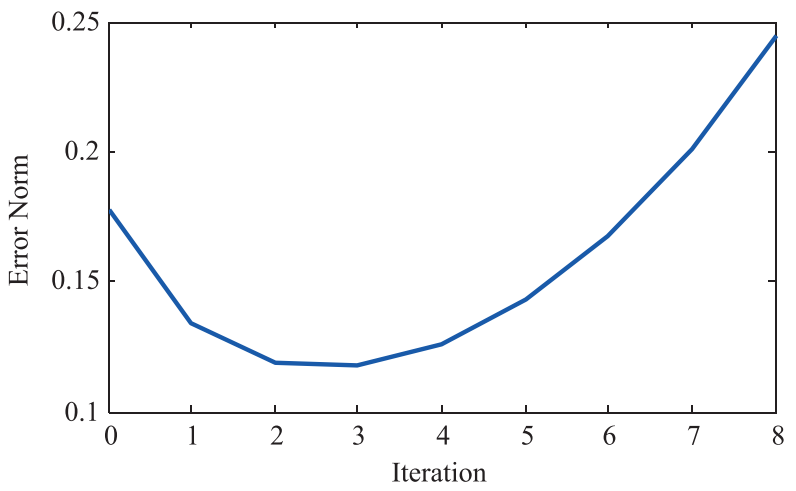

Fig. 5. Instability in setpoint tracking without disturbance; the desired reference is $r(t)=1.0$. (a) Evolution of the output from the first to the last iteration and (b) Evolution of the error $\mathcal{L}_{2}$ norm in the iteration domain. [Color figure can be viewed at wileyonlinelibrary.com]

uncertainty is low at DC, the MRAILC system learns a large bandwidth that is necessary to compensate for the transient error in response to a step reference. In particular, we observe in Fig. 5 that the response to the learned input at the 8th iteration shows oscillatory behavior due to the high-gain operators, as opposed to the initial output which is devoid of high frequencies. We see a similar trend of initial steady decrease of the error for regulation in response to a load disturbance in Fig. 6, where the error starts to increase with each iteration after the 9th trial. Again, the high-gain operators result in instability despite the low bandwidth of the exogenous signals $r$ and $d$.

Next, we reconsider setpoint tracking at a lower amplitude, where $r(t)=0.1$, but assume a persistent sinusoidal disturbance given by $d(t)=0.01 \sin (8 t)$. Fig. 7 shows that the MRAILC algorithm performs well and reduces the tracking error this time for about 25 iterations, before beginning to diverge. In this case, the lower amplitude of the signals help maintain stability for a

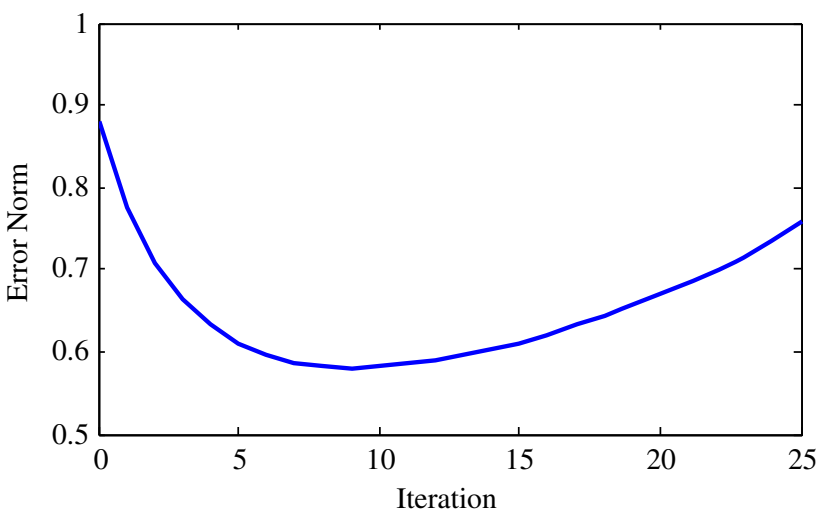

Fig. 6. Instability in regulation with a load disturbance; the desired reference is $r(t)=0.0$ and the disturbance is $d(t)=1.0$. [Color figure can be viewed at wileyonlinelibrary.com]

(a)

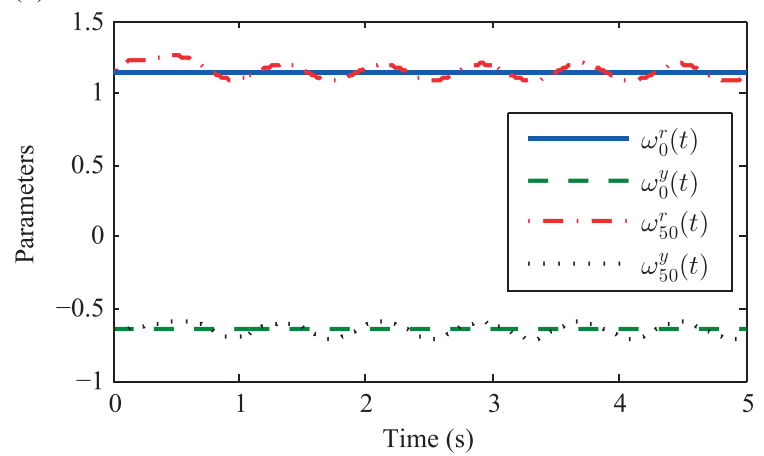

(b)

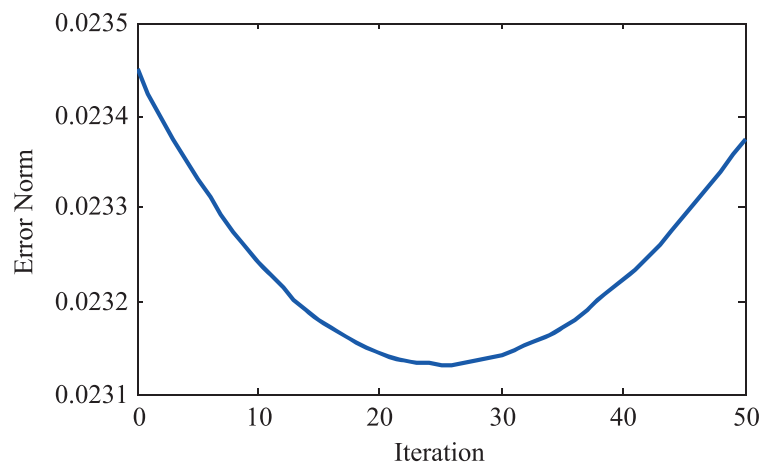

Fig. 7. Instability in setpoint tracking with a sinusoidal disturbance; the desired reference is $r(t)=0.1$ and the disturbance is $d(t)=0.01 \sin (8 t)$. (a) Evolution of the parameter vector from the first to the last iteration and (b) Evolution of the error $\mathcal{L}_{2}$ norm in the iteration domain. [Color figure can be viewed at wileyonlinelibrary.com] 


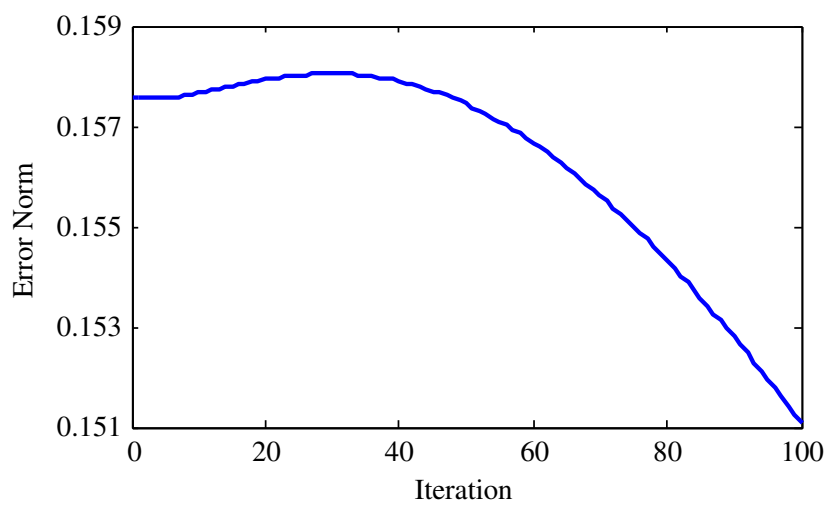

Fig. 8. Stable learning of setpoint tracking with sinusoidal disturbances at low amplitude and bandwidth; the desired reference is $r(t)=0.1$ and the disturbance is $d(t)=0.1 \sin (4 t)$. [Color figure can be viewed at wileyonlinelibrary.com]

longer time, but the algorithm cannot counteract the phase shift inherent in the system. A closer look at the evolution of the parameter vector shows that the adaptation law has roughly learned the frequency of the disturbance, so the phase error of the system must be the source of the problem that leads to parameter drift.

Finally, we note in Fig. 8 that when the disturbance is changed to $d(t)=0.1 \sin (4 t)$, the algorithm progressively decreases the error despite an initial transient phase. This implies that the exogenous signals have to have a low enough frequency and amplitude to preserve stability and prevent bursting: In this case, it seems that for setpoint tracking at $r(t)=0.1$, the upper bound for the frequency of admissible disturbances lies somewhere between 4 and $8 \mathrm{rad} / \mathrm{s}$, while the amplitude can possibly be greater than 0.1 .

\section{CONCLUSION}

In this paper, we studied the robustness problem of AILC in the presence of unmodeled dynamics. We generalized function space concepts to ILC systems to define the notion of gain and showed the existence of infinite-gain operators in an MRAILC algorithm. Heuristic linear systems arguments were put forth to explain how instability can occur, which were backed by several simulation examples. Our findings indicate that the shortcomings of MRAILC in terms of robustness are no different than those of MRAC. In fact, the robustness issue is found to be more severe with certain cases demonstrating that the learning operation deteriorates a readily satisfactory tracking performance given by MRAC.
It is reasonable to say that similar problems can occur in a variety of AILC schemes. As a matter of fact, most ILC systems that achieve perfect tracking are essentially iterative integrators [7] and are prone to instability in the presence of unmodeled dynamics. For example, stability of proportional-derivative type ILC schemes depend on a specific relative degree assumption [2]. However, in the nonadaptive case, there are easy remedies for this such as the use of the low-pass $Q$-filter $[8,12]$. In the adaptive feedback case, although the parameter drift problem has been resolved, the ability to adjust closed-loop bandwidth is a nontrivial problem [14]. In general, filtering has been shown not to be effective and even detrimental, save for some recent approaches given in $[9,24]$. Based on the similarities between adaptive feedback control and AILC, similar results are likely to be encountered in the iterative case. We believe further research is necessary to investigate these issues and understand how AILC can be made to be more robust under uncertainties.

\section{REFERENCES}

1. Ahn, H.-S., Y.-Q. Chen, and K. L. Moore, "Iterative learning control: Brief survey and categorization," IEEE Trans. Syst. Man Cybern. C: Applicat. Rev, Vol. 37, No. 6, pp. 1099-1121 (2007).

2. Altın, B. and K. Barton, "Exponential stability of nonlinear differential repetitive processes with applications to iterative learning control," Automatica, Vol. 81, pp. 369-376 (2017).

3. Anderson, B. D. O., "Failures of adaptive control theory and their resolution," Commun. Inform. Syst., Vol. 5, No. 1, pp. 1-20 (2005).

4. Astrom, K., "A commentary on the C.E. Rohrs et al. paper "robustness of continuous-time adaptive control algorithms in the presence of unmodeled dynamics"," IEEE Trans. Autom. Control, Vol. 30, No. 9, pp. 889-889 (1985).

5. Astrom, K. J., "Analysis of Rohrs counterexamples to adaptive control," Vol. 22, 22nd IEEE Conf. Decis. Control, San Antonio, TX, USA, pp. 982-987 (1983).

6. Astrom, K. J., "Interactions between excitation and unmodeled dynamics in adaptive control," Vol. 23, 23rd IEEE Conf. Decis. Control, Las Vegas, NV, USA, pp. 1276-1281 (1984).

7. Bristow, D. A., M. Tharayil, and A. G. Alleyne, "A survey of iterative learning control," IEEE Control Syst., Vol. 26, No. 3, pp. 96-114 (2006).

8. Bristow, D. A., K. L. Barton, and A. G. Alleyne, "Iterative learning control," In Levine, W. S. (Ed.) The Control Handbook, CRC Press, Salem, MA (2010). 
9. Cao, C. and N. Hovakimyan, $\mathcal{L}_{1}$ Adaptive Control Theory: Guaranteed Robustness with Fast Adaptation, Society for Industrial and Applied Mathematics, Philadelphia, PA (2010).

10. Chien, C.-J. and A. Tayebi, "Further results on adaptive iterative learning control of robot manipulators," Automatica, Vol. 44, No. 3, pp. 830 -837 (2008).

11. Chien, C.-J. and C.-Y. Yao, "Iterative learning of model reference adaptive controller for uncertain nonlinear systems with only output measurement," Automatica, Vol. 40, No. 5, pp. 855-864 (2004).

12. De Roover, D. and O. H. Bosgra, "Synthesis of robust multivariable iterative learning controllers with application to a wafer stage motion system," Int. J. Control, Vol. 73, No. 10, pp. 968-979 (2000).

13. French, M. and E. Rogers, "Non-linear iterative learning by an adaptive Lyapunov technique," Int. J. Control, Vol. 73, No. 10, pp. 840-850 (2000).

14. Ioannou, P. A. and J. Sun, Robust Adaptive Control, Prentice-Hall, Upper Saddle River, NJ (1996).

15. Ortega, R. and Y. Tang, "Robustness of adaptive controllers-A survey," Automatica, Vol. 25, No. 5, pp. $651-677$ (1989).

16. Owens, D. H. and G. Munde, "Error convergence in an adaptive iterative learning controller," Int. J. Control, Vol. 73, No. 10, pp. 851-857 (2000).

17. Rohrs, C. E., L. Valavani, M. Athans, and G. Stein, "Robustness of continuous-time adaptive control algorithms in the presence of unmodeled dynamics," IEEE Trans. Autom. Control, Vol. 30, No. 9, pp. 881-889 (1985).

18. Sun, D. and J. K. Mills, "High-accuracy trajectory tracking of industrial robot manipulator using adaptive-learning scheme," Vol. 3, Proc. Amer. Control Conf., San Diego, CA, USA, pp. 1935-1939 (1999).

19. Tayebi, A., "Model reference adaptive iterative learning control for linear systems," Int. J. Adapt. Control Signal Process., Vol. 20, No. 9, pp. 475-489 (2006).

20. Tayebi, A., "Adaptive iterative learning control for robot manipulators," Automatica, Vol. 40, No. 7, pp. $1195-1203$ (2004).

21. Tian, Y.-P. and X. Yu, "Robust learning control for a class of nonlinear systems with periodic and aperiodic uncertainties," Automatica, Vol. 39, No. 11, pp. 1957-1966 (2003).

22. Xu, J.-X., "A survey on iterative learning control for nonlinear systems," Int. J. Control, Vol. 84, No. 7, pp. 1275-1294 (2011).

23. Xu, J.-X. and B. Viswanathan, "Adaptive robust iterative learning control with dead zone scheme," Automatica, Vol. 36, No. 1, pp. 91-99 (2000).

24. Yucelen, T., G. De La Torre, and E. N. Johnson, "Frequency-limited adaptive control architecture for transient response improvement," Amer. Control Conf., Washington, DC, USA, pp. 6631-6636 (2013).

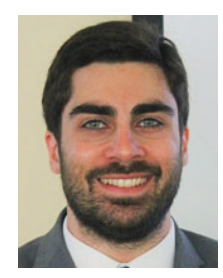

Berk Altın received his BS in Mechatronics from Sabancı University, Istanbul, Turkey, in 2011. From 2011 to 2016, he attended the University of Michigan, Ann Arbor, as a Fulbright fellow, where he received the M.S. and Ph.D. degrees in Electrical Engineering: Systems, and the M.S. degree in Mathematics, in 2013, 2016 and 2016, respectively. $\mathrm{He}$ is currently employed as a postdoctoral researcher at the University of California, Santa Cruz, with the Hybrid Systems Laboratory. His primary research interests include hybrid systems, model predictive control, iterative learning control, repetitive processes, and multidimensional systems, with applications in cyberphysical systems, robotics, and additive manufacturing.

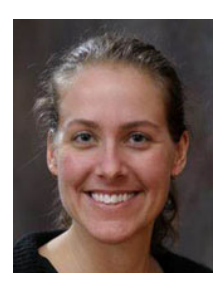

Kira Barton is an Associate Professor and Miller Faculty Scholar in the Department of Mechanical Engineering at the University of Michigan. She received her B.Sc. in Mechanical Engineering from the University of Colorado at Boulder in 2001. She continued her education in mechanical engineering at the University of Illinois at Urbana-Champaign and completed her M.Sc. and Ph.D. degrees in 2006 and 2010, respectively. She held a postdoctoral research position at the University of Illinois from Fall 2010 until Fall 2011, at which point she joined the Mechanical Engineering Department at the University of Michigan at Ann Arbor. Kira conducts research in modeling, sensing, and control for applications in advanced manufacturing and robotics, with specializations in iterative learning control, smart manufacturing and micro-additive manufacturing. Kira is the recipient of an NSF CAREER Award in 2014, 2015 SME Outstanding Young Manufacturing Engineer Award, the 2015 University of Illinois, Department of Mechanical Science and Engineering Outstanding Young Alumni Award, the 2016 University of Michigan, Department of Mechanical Engineering Department Achievement Award, and the 2017 ASME Dynamic Systems and Control Young Investigator Award. 